Supporting Information

\title{
Examining the Ground and First Excited States of Methyl Peroxy Radical with High-Level Coupled-Cluster Theory
}

\author{
Andreas V. Copan \\ Center for Computational Quantum Chemistry \\ University of Georgia, Athens, GA 30602 \\ Henry F. Schaefer III \\ Center for Computational Quantum Chemistry \\ University of Georgia, Athens, GA 30602 \\ Jay Agarwal* \\ Center for Computational Quantum Chemistry \\ University of Georgia, Athens, GA 30602 \\ jagarwal@uga.edu
}




\section{Contents}

1 Data for Stationary Points $\quad$ S1

$1.1 \mathrm{CH}_{3} \mathrm{O}_{2}\left(\tilde{X}^{2} A^{\prime \prime}\right) \ldots \ldots \ldots \ldots \ldots \ldots$. . . . . . . . . . . . . . . . . . .

1.1.1 CCSD $(\mathrm{T}) / \mathrm{ANO} 2$ Optimization and Frequencies . . . . . . . . . . S1

1.1.2 $\mathrm{CCSD}(\mathrm{T}) / \mathrm{ANO} 1$ Optimization and Frequencies . . . . . . . . . S2

1.1.3 BCCD $(\mathrm{T}) / \mathrm{ANO} 1$ Optimization and Frequencies . . . . . . . . . . S3

1.1.4 Anharmonicity Constants and ZPVE $[\mathrm{CCSD}(\mathrm{T}) / \mathrm{ANO} 1]$. . . . . . . S S4

$1.2 \mathrm{CH}_{3} \mathrm{O}_{2}\left(\tilde{A}^{2} A^{\prime}\right) \ldots \ldots \ldots \ldots \ldots \ldots \ldots$

1.2.1 CCSD $(\mathrm{T}) / \mathrm{ANO} 2$ Optimization and Frequencies . . . . . . . . . . S5

1.2.2 CCSD $(\mathrm{T}) / \mathrm{ANO} 1$ Optimization and Frequencies . . . . . . . . . . S6

1.2.3 BCCD $(\mathrm{T}) / \mathrm{ANO} 1$ Optimization and Frequencies . . . . . . . . . . S7

1.2.4 Anharmonicity Constants and ZPVE $[\mathrm{CCSD}(\mathrm{T}) / \mathrm{ANO} 1] \ldots$. . . . . S8 


\section{Data for Stationary Points}

\section{$1.1 \mathrm{CH}_{3} \mathrm{O}_{2}\left(\tilde{X}^{2} A^{\prime \prime}\right)$}

\subsubsection{CCSD(T)/ANO2 Optimization and Frequencies}

Software:

Theory:

Reference:

CC Convergence:

SCF Convergence:

Charge:

Multiplicity:

Frozen Core:

Geometry Convergence:

Equilibrium Geometry $(\AA)$ :

Harmonic Frequencies $\left(\mathrm{cm}^{-1}\right)$ :

$\begin{array}{crr}\text { H } & -1.9 \\ \text { C } & -1.1 \\ 0 & 0.0 \\ 0 & 1.096 \\ \text { H } & -1.1 \\ \text { Harmonic Frequencies }\left(\mathrm{cm}^{-1}\right): & & \\ & & \\ & 150.4895 \\ & 494.4092 \\ & 947.4901 \\ & 1145.7432 \\ & 1167.7365 \\ & 1218.7031 \\ & 1450.3407 \\ 1483.9004 \\ 1496.9575 \\ 3063.8861 \\ 3165.6327 \\ 3174.9126\end{array}$

$\mathrm{UHF}$

$10^{-10}$

$10^{-10}$

0

2

$\mathrm{ON}$

150.4895

494.4092

97.4901

1145.7432

1167.7365

(8.7031

1450.3407

1483.9004

9575

3165.6327

3174.9126
$\operatorname{CCSD}(\mathrm{T}) / \mathrm{ANO} 2$

$10^{-9} \mathrm{H} /$ bohr

$\begin{array}{rrrr}\mathrm{H} & -1.9715478549 & 0.4931916402 & 0.0000000000 \\ \mathrm{C} & -1.1662002125 & -0.2372485708 & -0.0000000000 \\ \mathrm{O} & 0.0535785297 & 0.5312531795 & 0.0000000000 \\ \mathrm{O} & 1.0961886549 & -0.2767437253 & -0.0000000000 \\ \mathrm{H} & -1.1951727886 & -0.8537816537 & 0.8954106256 \\ \mathrm{H} & -1.1951727886 & -0.8537816537 & -0.8954106256\end{array}$




\subsubsection{CCSD(T)/ANO1 Optimization and Frequencies}

Software:

Theory:

Reference:

CC Convergence:

SCF Convergence:

Charge:

Multiplicity:

Frozen Core:

Geometry Convergence:

Equilibrium Geometry $(\AA)$ :
CFour 1.0

$\operatorname{CCSD}(\mathrm{T}) / \mathrm{ANO} 1$

$\mathrm{UHF}$

$10^{-10}$

$10^{-10}$

0

2

$\mathrm{ON}$

$10^{-9} \mathrm{H} /$ bohr

Harmonic Frequencies $\left(\mathrm{cm}^{-1}\right)$ :

$\begin{array}{rrrr}\mathrm{H} & -1.9757680011 & 0.4896441682 & -0.0000000000 \\ \mathrm{C} & -1.1671412220 & -0.2389183419 & -0.0000000000 \\ \mathrm{O} & 0.0519764191 & 0.5340179401 & -0.0000000000 \\ \mathrm{O} & 1.0990018882 & -0.2777022346 & 0.0000000000 \\ \mathrm{H} & -1.1970712120 & -0.8564002979 & 0.8960315357 \\ \mathrm{H} & -1.1970712120 & -0.8564002979 & -0.8960315357\end{array}$

152.7290

493.2919

945.4622

1143.7668

1157.5911

1212.2737

1446.9509

1480.1304

1493.3824

3061.2660

3162.3232

3170.5454 


\subsubsection{BCCD(T)/ANO1 Optimization and Frequencies}

Software:

Theory:

Reference:

CC Convergence:

SCF Convergence:

Brueckner Convergence:

Charge:

Multiplicity:

Frozen Core:

Geometry Convergence:

Equilibrium Geometry $(\AA)$ :
PSI 4

$\operatorname{BCCD}(\mathrm{T}) / \mathrm{ANO} 1$

Brueckner

$10^{-9}$

$10^{-11}$

$10^{-8}$

0

2

$\mathrm{ON}$

$10^{-6} \mathrm{H} / \mathrm{bohr}$

Harmonic Frequencies $\left(\mathrm{cm}^{-1}\right)$ :

$\begin{array}{rrrr}\text { H } & -0.958966682798 & 1.795791386988 & 0.000000000000 \\ \text { C } & -0.958674160368 & 0.707297498933 & 0.000000000000 \\ \text { O } & 0.431632483290 & 0.319472808828 & 0.000000000000 \\ \text { O } & 0.529153839342 & -1.003085199354 & 0.000000000000 \\ \text { H } & -1.437320245925 & 0.315981593870 & 0.896048100959 \\ \text { H } & -1.437320245925 & 0.315981593870 & -0.896048100959\end{array}$

153.439

492.694

945.041

1143.163

1150.704

1210.868

1446.300

1479.723

1492.921

3060.747

3162.103

3170.082 


\subsubsection{Anharmonicity Constants and ZPVE $[\mathrm{CCSD}(\mathrm{T}) / \mathrm{ANO} 1]$}

$$
\begin{aligned}
& \mathrm{x}_{-} 1,1=-9.66884 \\
& x_{-} 2,2=0.19028 \\
& \mathrm{x}_{-} 3,3=-5.83896 \\
& x_{-} 4,4=-2.03363 \\
& \mathrm{x}_{-} 5,5=-6.89569 \\
& \mathrm{x}_{-} 6,6=-1.86821 \\
& \mathrm{x}_{-} 1,2=11.88161 \\
& x_{-} 1,3=-2.26047 \\
& \mathrm{x}_{-} 1,4=1.40740 \\
& \mathrm{x}_{-} 1,5=-1.42359 \\
& x_{-} 1,6=-5.97060 \\
& x_{-} 1,7=-1.54107 \\
& \mathrm{x}_{-} 1,8=-1.31895 \\
& \mathrm{x}_{-} 1,9=-1.91022 \\
& \mathrm{x}_{-} 1,10=-0.21871 \\
& \mathrm{x}_{-} 1,11=0.29112 \\
& \mathrm{x}_{-} 1,12=-1.84458 \\
& \mathrm{x}_{-} 2,3=-7.55780 \\
& \mathrm{x}_{-} 2,4=0.04199 \\
& \mathrm{x}_{-} 2,5=-4.76345 \\
& \mathrm{x}_{2} 2,6=-1.79602 \\
& \mathrm{x}_{2} 2,7=1.39187 \\
& \mathrm{x}_{-} 2,8=-1.42306 \\
& x_{-} 2,9=0.09219 \\
& \mathrm{x}_{-} 2,10=-0.06486 \\
& \mathrm{x}_{-} 2,11=0.39065 \\
& \mathrm{x}_{-} 2,12=-0.61208 \\
& \mathrm{x}_{-} 3,4=-7.52320 \\
& x_{-} 3,5=-9.42528 \\
& \mathrm{x}_{-} 3,6=-6.89756 \\
& \mathrm{x}_{-} 3,7=-4.86275 \\
& \mathrm{x}_{-} 3,8=-1.90266 \\
& \mathrm{x}_{-} 3,9=-2.59308 \\
& \mathrm{x}_{-} 3,10=0.71644 \\
& \mathrm{x}_{-} 3,11=3.11008 \\
& \mathrm{x}_{-} 3,12=0.74961 \\
& \mathrm{x}_{-} 4,5=-2.82357 \\
& x_{-} 4,6=-3.32097 \\
& \mathrm{x}_{-} 4,7=-5.74170
\end{aligned}
$$

$$
\begin{aligned}
& \mathrm{x}_{-} 7,7=-8.32992 \\
& \mathrm{x}_{-} 8,8=-6.86582 \\
& \mathrm{x}_{-} 9,9=-8.08746 \\
& \mathrm{x}_{-} 10,10=-18.08354 \\
& \mathrm{x}_{-} 11,11=-33.00780 \\
& \mathrm{x}_{-} 12,12=-34.25648 \\
& \mathrm{x}_{-} 4,8=-7.67892 \\
& \mathrm{x}_{-} 4,9=-7.14413 \\
& \mathrm{x}_{-} 4,10=-4.69150 \\
& \mathrm{x}_{-} 4,11=-5.64316 \\
& \mathrm{x}_{-} 4,12=-4.63702 \\
& \mathrm{x}_{-} 5,6=-9.31200 \\
& \mathrm{x}_{-} 5,7=-4.72378 \\
& \mathrm{x}_{-} 5,8=-1.48031 \\
& \mathrm{x}_{-} 5,9=-1.26340 \\
& \mathrm{x}_{-} 5,10=-0.82784 \\
& \mathrm{x}_{-} 5,11=-0.38546 \\
& x_{-} 5,12=-1.40267 \\
& \mathrm{x}_{-} 6,7=-5.13684 \\
& \mathrm{x}_{-} 6,8=-4.40995 \\
& \mathrm{x}_{-} 6,9=-4.59645 \\
& \mathrm{x}_{-} 6,10=-3.83133 \\
& \mathrm{x}_{-} 6,11=-4.63960 \\
& \mathrm{x}_{-} 6,12=-5.34873 \\
& \mathrm{x}_{-} 7,8=-2.56299 \\
& \mathrm{x}_{-} 7,9=-2.88078 \\
& \mathrm{x}_{-} 7,10=13.96527 \\
& \mathrm{x}_{-} 7,11=-8.86739 \\
& \mathrm{x}_{-} 7,12=-12.02197 \\
& x_{-} 8,9=-4.35688 \\
& \mathrm{x}_{-} 8,10=-2.42817 \\
& \mathrm{x}_{-} 8,11=-11.33774 \\
& \mathrm{x}_{-} 8,12=-19.80296 \\
& \mathrm{x}_{-} 9,10=4.52126 \\
& \mathrm{x}_{-} 9,11=-20.47045 \\
& \mathrm{x}_{-} 9,12=-8.81011 \\
& \mathrm{x}_{-} 10,11=-81.08893 \\
& \mathrm{x}_{-} 10,12=-68.91635 \\
& \mathrm{x}_{-} 11,12=-35.74187
\end{aligned}
$$

Anharmonic ZPVE: $\quad 9324.31320 \mathrm{~cm}-1 \quad 26.65956 \mathrm{kcal} / \mathrm{mol}$

$$
\begin{array}{rr}
\text { Quadratic }= & 9459.85649 \mathrm{~cm}-1 \\
\text { Cubic }= & -23.01921 \mathrm{~cm}-1 \\
\text { Quartic }= & -202.55957 \mathrm{~cm}-1 \\
\mathrm{Z} \text { kinetic }= & 84.82734 \mathrm{~cm}-1
\end{array}
$$




\section{$1.2 \quad \mathrm{CH}_{3} \mathrm{O}_{2}\left(\tilde{A}^{2} \boldsymbol{A}^{\prime}\right)$}

\subsubsection{CCSD(T)/ANO2 Optimization and Frequencies}

Software:

Theory:

Reference:

CFOur 1.0

CC Convergence:

$\operatorname{CCSD}(\mathrm{T}) / \mathrm{ANO} 2$

$\mathrm{UHF}$

SCF Convergence:

$10^{-10}$

Charge:

$10^{-10}$

Multiplicity:

Frozen Core:

0

Geometry Convergence:

Equilibrium Geometry $(\AA)$ :

Harmonic Frequencies $\left(\mathrm{cm}^{-1}\right)$ :

2

$\mathrm{ON}$

$10^{-9} \mathrm{H} / \mathrm{bohr}$

$\begin{array}{rrrr}\text { H } & -1.9768010768 & 0.4169999749 & -0.0000000000 \\ \mathrm{C} & -1.1362995072 & -0.2719180839 & -0.0000000000 \\ \mathrm{O} & 0.0152154969 & 0.5775828222 & -0.0000000000 \\ \mathrm{O} & 1.1069259259 & -0.2873997041 & 0.0000000000 \\ \mathrm{H} & -1.1513370365 & -0.8923673166 & 0.8967847719 \\ \mathrm{H} & -1.1513370365 & -0.8923673166 & -0.8967847719\end{array}$

248.8533

384.6010

919.3981

1040.0474

1177.8649

1185.6792

1454.3644

1478.5819

1519.5214

3041.2087

3122.3878

3167.9767 


\subsection{2 $\operatorname{CCSD}(\mathrm{T}) / \mathrm{ANO} 1$ Optimization and Frequencies}

Software:

Theory:

Reference:

CC Convergence:

SCF Convergence:

Charge:

Multiplicity:

Frozen Core:

Geometry Convergence:

Equilibrium Geometry $(\AA)$ :

Harmonic Frequencies $\left(\mathrm{cm}^{-1}\right)$ :
CFour 1.0

CCSD(T)/ANO1

UHF

$10^{-10}$

$10^{-10}$

0

2

ON

$10^{-9} \mathrm{H} /$ bohr
248.7078
385.0024
909.5586
1038.9391
1176.3538
1183.3745
1451.2102
1474.2561
1516.9632
3037.9148
3117.7529
3164.2166 


\subsection{3 $\mathrm{BCCD}(\mathrm{T}) / \mathrm{ANO} 1$ Optimization and Frequencies}

Software:

Theory:

Reference:

CC Convergence:

SCF Convergence:

Brueckner Convergence:

Charge:

Multiplicity:

Frozen Core:

Geometry Convergence:

Equilibrium Geometry $(\AA)$ :

Harmonic Frequencies $\left(\mathrm{cm}^{-1}\right)$ :

PSI 4

$\mathrm{BCCD}(\mathrm{T}) / \mathrm{ANO} 1$

Brueckner

$10^{-9}$

$10^{-11}$

$10^{-8}$

0

2

ON

$10^{-6} \mathrm{H} / \mathrm{bohr}$

H -1.9819103880

C $-1.1373832421-0.2744211720$

$\begin{array}{llll}0 & 0.0117287415 & 0.5804260369\end{array}$

$\mathrm{H}-1.1525600178-0.8962725981 \quad 0.8973184818$

H $-1.1525600178-0.8962725981-0.8973184818$ 


\subsubsection{Anharmonicity Constants and ZPVE [CCSD(T)/ANO1]}

$$
\begin{aligned}
& \mathrm{x}_{-} 1,1=-6.52892 \\
& x_{-} 2,2=0.32996 \\
& \mathrm{x}_{-} 3,3=1.25366 \\
& \mathrm{x}_{-} 4,4=-7.66389 \\
& \mathrm{x}_{-} 5,5=-2.00752 \\
& \mathrm{x}_{-} 6,6=-1.88322 \\
& \mathrm{x}_{-} 1,2=0.14619 \\
& \mathrm{x}_{-} 1,3=-1.12919 \\
& \mathrm{x}_{-} 1,4=-1.02279 \\
& x_{-} 1,5=-3.06804 \\
& \mathrm{x}_{-} 1,6=-0.96704 \\
& \mathrm{x}_{-} 1,7=1.29164 \\
& \mathrm{x}_{-} 1,8=2.31689 \\
& \mathrm{x}_{-} 1,9=-4.48388 \\
& \mathrm{x}_{-} 1,10=-0.37090 \\
& \mathrm{x}_{-} 1,11=1.71516 \\
& \mathrm{x}_{-} 1,12=-2.40973 \\
& \mathrm{x}_{-} 2,3=-3.23193 \\
& \mathrm{x}_{-} 2,4=-3.30417 \\
& \mathrm{x}_{-} 2,5=1.21225 \\
& \mathrm{x}_{-} 2,6=-0.79993 \\
& \mathrm{x}_{-} 2,7=-0.21917 \\
& \mathrm{x}_{-} 2,8=-1.57263 \\
& \mathrm{x}_{-} 2,9=-1.43488 \\
& \mathrm{x}_{-} 2,10=-0.06885 \\
& \mathrm{x}_{-} 2,11=0.20747 \\
& \mathrm{x}_{-} 2,12=-0.81378 \\
& \mathrm{x}_{-} 3,4=-0.31722 \\
& \mathrm{x}_{-} 3,5=-0.79005 \\
& \mathrm{x}_{-} 3,6=-0.55075 \\
& \mathrm{x}_{-} 3,7=-1.35526 \\
& \mathrm{x} \_3,8=-0.42799 \\
& \mathrm{x}_{-} 3,9=-0.84182 \\
& \mathrm{x}_{-} 3,10=-0.02144 \\
& \mathrm{x}_{-} 3,11=0.29465 \\
& \mathrm{x}_{-} 3,12=-1.32001 \\
& \mathrm{x}_{-} 4,5=-9.58357 \\
& x_{-} 4,6=-8.30829 \\
& \mathrm{x}_{-} 4,7=-7.20543
\end{aligned}
$$

$$
\begin{aligned}
& \mathrm{x}_{-} 7,7=-8.78697 \\
& \mathrm{x}_{-} 8,8=-6.18545 \\
& \mathrm{x}_{-} 9,9=-1.71235 *
\end{aligned}
$$$$
\mathrm{x}_{-} 10,10=-21.06183
$$$$
\mathrm{x}_{-} 11,11=-34.11702
$$$$
\mathrm{x}_{-} 12,12=-44.31269
$$$$
\mathrm{x}_{-} 4,8=-2.18160
$$$$
\mathrm{x}_{-} 4,9=-3.36734
$$$$
\mathrm{x}_{-} 4,10=2.19206
$$$$
\mathrm{x}_{-} 4,11=4.25092
$$$$
\mathrm{x}_{-} 4,12=1.12998
$$$$
\mathrm{x}_{-} 5,6=-0.16358
$$$$
\mathrm{x}_{-} 5,7=-4.51672
$$$$
\mathrm{x}_{-} 5,8=-6.60349
$$$$
\mathrm{x}_{-} 5,9=-11.23674
$$$$
\mathrm{x}_{-} 5,10=-5.79357
$$$$
\mathrm{x}_{-} 5,11=-4.01065
$$$$
x_{-} 5,12=-2.77230
$$$$
\mathrm{x}_{-} 6,7=-8.16560
$$$$
\mathrm{x}_{-} 6,8=-5.25823
$$$$
\mathrm{x}_{-} 6,9=-4.84766
$$$$
\mathrm{x}_{-} 6,10=-2.65264
$$$$
\mathrm{x}_{-} 6,11=-3.68611
$$$$
\mathrm{x}_{-} 6,12=-8.06699
$$$$
\mathrm{x}_{-} 7,8=-2.09348
$$$$
\mathrm{x}_{-} 7,9=-3.26014
$$$$
\mathrm{x}_{-} 7,10=17.10550
$$$$
\mathrm{x}_{-} 7,11=-8.61476
$$$$
\mathrm{x}_{-} 7,12=-13.73762
$$$$
\mathrm{x}_{-} 8,9=-5.01065
$$$$
\mathrm{x}_{-} 8,10=-3.60288
$$$$
\mathrm{x}_{-} 8,11=-10.23797
$$$$
\mathrm{x}_{-} 8,12=-19.72710
$$$$
\mathrm{x}_{-} 9,10=-22.69884 *
$$$$
\mathrm{x}_{-} 9,11=-18.36892
$$$$
\mathrm{x}_{-} 9,12=-5.33467
$$$$
\mathrm{x}_{-} 10,11=-99.82969
$$$$
\mathrm{x}_{-} 10,12=-44.57930
$$$$
\mathrm{x}_{-} 11,12=-19.16732
$$

*Near-zero denominators removed

Anharmonic ZPVE: $\quad 9220.89658 \mathrm{~cm}-1 \quad 26.36387 \mathrm{kcal} / \mathrm{mol}$ 